\title{
Therapeutic possibilities of plasmonically heated gold nanoparticles
}

\author{
Dakrong Pissuwan ${ }^{1}$, Stella M. Valenzuela ${ }^{1,2}$ and Michael B. Cortie ${ }^{1, *}$ \\ ${ }^{1}$ Institute for Nanoscale Technology, University of Technology Sydney, Broadway \\ NSW 2007, Australia \\ ${ }^{2}$ Dept. of Health Sciences, University of Technology Sydney, Broadway NSW 2007, \\ Australia
}

\begin{abstract}
Nanoparticles of gold, which are in the size range of 10 to $100 \mathrm{~nm}$, undergo a plasmon resonance with light. This is a process whereby the electrons of the gold resonate with incoming radiation causing them to both absorb and scatter light. The effect can be harnessed to either destroy tissue by local heating or release payload molecules of therapeutic importance. Gold nanoparticles can also be conjugated with biologically-active moities, providing possibilities for targeting to particular tissues. Here we review progress in the exploitation of this plasmon resonance of gold nanoparticles in photo-thermal therapeutic medicine.
\end{abstract}

\section{Introduction}

Metallic gold, either in the form of bulk surfaces or as nanoparticles, is widely used in the emerging and highly interdisciplinary field of nanotechnology [1-3]. Many biodiagnostic applications of gold nanoparticles or electrodes have been developed since the 1970s [4-10]. However, the rational application of gold nanoparticles in therapeutic situations is a largely undeveloped field. Two properties of gold nanoparticles are attractive in this context. Firstly, antibodies and other biological molecules can be readily attached to the surface of gold nanoparticles, and secondly the plasmon resonances of gold nanoparticles of certain shapes cause them to have photon capture cross sections that are four to five orders of magnitude greater than those of photothermal dyes [11]. These attributes provide the possibility of obtaining very localized heating or drug release in a therapeutic application.

Excellent reviews of the basic physical, chemical and optical properties of gold nanoparticles and their other applications are available $[1,12]$ and the medical uses of soluble gold compounds have been discussed by Shaw [13]. The medical applications of nanoparticles in general have been reviewed by Salata [14], while Moghimi et al. [15] have reviewed the use of nanoparticles in drug delivery schemes not involving light. Finally, the use of light to control drug delivery in liposomal systems was summarized by Shum [16]. However, while the medical applications of one kind of gold particle, the 'nanoshell' have been quite widely discussed recently [17-19], no broad synthesis of the recent developments in the photo-thermal, therapeutic possibilities of gold nanoparticles seems to exist in the literature at this time. This is the topic we address here, with only the most pertinent of the background information repeated.

\footnotetext{
* Corresponding author : Cortie M.B. (michael.cortie@uts.edu.au)
} 


\section{Relevant properties of gold nanoparticles}

The reader is referred to Pyykko for a recent overview of the chemical physics of gold [20]. The aspects that are most relevant here are gold's resistance to oxidation and its plasmon resonance with light. The latter is at $\sim 520 \mathrm{~nm}$ for ordinary gold nanospheres, in the middle of the visible spectrum, but can be red-shifted into the near infrared (NIR, 800-1200 nm) for more complex shapes such as nanorods - see Box 1. This is useful because body tissue is reasonably transparent to NIR light $[11,17,21]$, providing a window of sorts into the body by a number of methods.

Gold or silver metallo-dielectric core-shell particles offer another route to spectral control of both absorption and scattering of light, particularly if the core is a dielectric (most commonly gold sulfide, silica or polystyrene) and the shell is a metal. The spectral properties of such shapes can be controlled by varying the relative dimensions of the core and shell [19, 22-27], Figure 1. Several groups have reported successful recipes for the synthesis of these shapes, with the group of Halas at Rice University leading the way with a number of papers [9, 11, 17-19, 21, 27-33] and patents [34-38] on the manufacture and potential medical applications of these particles. Although the term 'nanoshell' is extant in North America for these particles, the term 'core-shell particle' is often used in Europe [39,40] for both metal-ondielectric, and dielectric-on-metal particles.

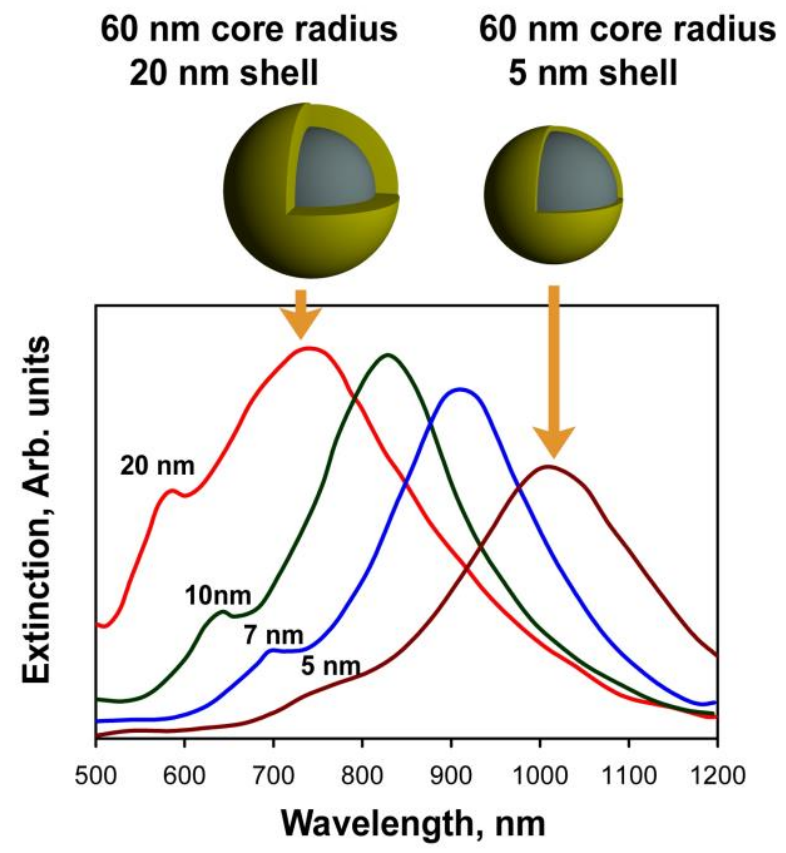

Figure 1. The optical response of gold nano-shells at different sizes, showing how the extinction peak is red-shifted for relatively thinner shells (adapted and redrawn with permission from Loo, C. et al. "Nanoshell-enabled photonics-based imaging and therapy of cancer:, TCRT 3, (2004) 33-40, www.tcrt.com). 


\section{Biomedical exploitation of gold nanoparticles}

\section{Targeting strategies for nanoparticles}

A disadvantage of many drug-based therapies (particularly when used orally or intravenously) is that the introduced compound is not localized to the target site, but is widely dispersed. Treatments based on nanoparticles can improve the situation. While the body can concentrate inert nanoparticles by 'passive' targeting, the therapeutic delivery system can enhanced by functionalizing the surface of the gold particle so that it only targets specific tissues by 'active' targeting [15]. The two scenarios are illustrated in Figure 2 and further compared in the text below.

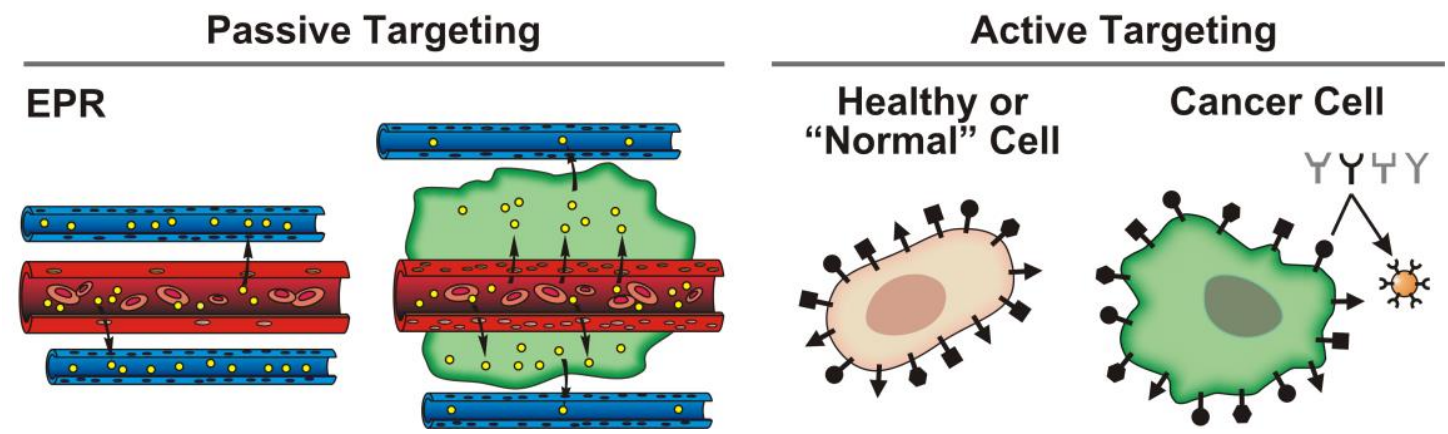

Figure 2. Passive and active targeting of nanoparticles showing passive targeting of a tumor by extravasation (left) and active targeting of a carcinoma by an antibody-functionalized nanoparticle (right) (redrawn from McNeil [58] with permission).

\section{Passive targeting}

Nanoparticle colloids can be designed so that the particles are just big enough to be retained in the liver and spleen while passing relatively freely though other organs [15]. This type of passive targeting has been used in radiotherapy of liver cancers, since the sinus endothelium of that organ has openings of $150 \mathrm{~nm}$ in diameter, while the spleen filters out particles greater than $250 \mathrm{~nm}$ in size [15]. Similarly, the fact that the vasculature of tumors is somewhat more permeable that that of healthy issue [41] has also been exploited to concentrate nanoparticles. O'Neal et al reported that nanoshells with diameters of $130 \mathrm{~nm}$ would pass preferentially through the walls of vessels, and would be deposited and concentrated in the surrounding tumorous tissue [21]. This phenomenon of 'extravasation' provides another passive method to concentrate particles of up to $300 \mathrm{~nm}$ diameter in some tumors or inflamed tissues [15].

\section{Active targeting}

Reliance upon retention of nanoparticles by size-specific filtration has obvious limitations. A more sophisticated approach is to functionalize the surface of the nanoparticle with an antibody or ligand for the desired target. Of course, this approach also has limitations due to non-specific binding and potential activation of the normal host immune response. The problem of an interaction between nanoparticle and immune system can be substantially alleviated by so-called 'stealth' technologies, which involve coating the nanoparticle with a self-assembled layer of a thiolated PEG 
(poly-ethyleneglycol) [2,15,42]. The surfaces of such particles are reasonably inert with respect to protein absorption. Alternatively a liposome coating may have merit for the same reason. Of course, if a particle is so well coated that it becomes invisible to the immune system, then it will also probably lose its functionality in respect of binding to specific receptors.

Once the gold nanoparticle is concentrated in the desired location, it can be activated by absorption of radiation of an appropriate wavelength. Broadly speaking, this action could be designed to deliver one of two therapeutic payloads; the localized generation of heat, or the localized release of a chemical. However, it may also be helpful to use the attached gold particles to simultaneously track or image the cells $[9,11]$.

\section{Hyperthermal therapy}

Local application of heat is a well-known concept in therapeutic medicine, and has been extensively explored for the treatment of cancer or other conditions. Energizing sources such as infrared lamps, ultrasound, or lasers can be used in the process, but there is always the problem of limiting the heat generated to the region of the target tissue [43]. As mentioned earlier, the problem can be solved in part by using gold nanoparticles tuned to absorb in the NIR such that the resulting localized heating causes irreversible thermal cellular destruction [18,19]. Although dyes can and have been used for this purpose, particles such as gold nanoshells have absorption efficiencies that are many orders of magnitude greater and that are not effected by photobleaching [18, 21]. Furthermore, as we have seen, there are ways to target nanoparticles that are simply not available for pharmaceutical compounds solubilised in vivo.

The first account of the use of gold particles in hyperthermal therapy appeared in $2003[18,36]$. In this and follow-up work by the Halas group, gold-on-silica nanoshells were used to actively target breast carcinoma cells by using the HER2 antibody [11], while extravasation was used to concentrate PEG-sheathed gold-on-silica nanoshells in an in vivo murine model by passive targeting [21]. NIR irradiation was found in the latter study to raise the temperature of the target regions by 40 to $50^{\circ} \mathrm{C}$, which selectively destroyed the carcinomas. The survival rate of mice treated in this manner was excellent compared to controls.

It is interesting to note, however, that the optical absorption spectrum of the gold particles used in the above work (for example, Figure 1 in reference [18]) had a very broad peak, and did not correspond to that of a particularly well-defined dispersion of nanoshells. Actually spectra of this type are also readily obtained with aggregated dispersions of solid gold nanospheres, $c f$. [44]. Not surprisingly therefore, the therapeutic effect can be obtained with particle morphologies other than nanoshells. This has been elegantly shown by Pitsillides et al,[45] who selectively destroyed CD8+ lymphocytes in a mixed CD8+/CD8- lymphocyte culture using ordinary colloidal gold nanoparticles. The particles were conjugated with a CD8+-specific antibody and used together with a $532 \mathrm{~nm}$ laser, causing highly selective apoptosis of the CD8+ population. In addition, systematic variation of the experimental technique revealed conditions that selectively permeabilized the cell membranes of the target population for up to two minutes, coupled with only a modest lethality. This provides 
a unique and hitherto scarcely exploited therapeutic modality to access intracellular targets.

Finally, there is also an effect that causes enhanced absorption of X-ray radiation at the interface between materials of high and low atomic number. Although not an effect of plasmon resonance, the effect also causes a localized generation of heat and has the same net effect. For example, the injection of $1.9 \mathrm{~nm}$ gold nanoparticles into murine tumors, followed by x-ray irradiation, caused tumor destruction [46]. In common with others [47], these workers found that the excess gold nanoparticles were cleared by the kidneys and that they were not toxic to the mice.

\section{Drug delivery}

In this scenario the basic ideas are to encapsulate the payload inside a gold coreshell particle, or to impregnate a larger drug-containing nano- or microparticle with suitably designed gold particles, or to bind the payload to the outer surface of a gold nanoparticle. Once at the target, release of the payload is induced by plasmonic heating. In general, it is envisaged that the source of light would be a laser in order that the necessary precision of location and wavelength be achieved. Gold nanoparticles themselves are sufficiently small that in principle they might be capable of delivering the payload of therapeutic agent or heat, directly into the cytoplasm or nucleus of the target cell $[15,48,49]$.

The first mention of photo-activated drug release by plasmonically active particles seems to have been in 2000 [17,27] followed by the appearance of a related US patent a little later [35]. In both cases the work exploited a polymer gel impregnated with gold nanoshells. These gold nanoshells were gold-on-gold sulfide core-shell particles. This type of nanoshell was discovered by Zhou in 1994 [26] and so was not new, but the novelty was its incorporation into a polymer composite with the intention of securing controlled drug release.

More recently, the plasmonic photo-activation of hollow polymer capsules containing model drugs has been demonstrated. First, Skirtach and colleagues described a scheme in which silver nanoparticles were incorporated into hollow polyelectrolyte capsules, which could then be burst by the light of a laser [50]. Evidently the effect of the illumination is to heat up the silver nanoparticles causing the polymer capsules to split and release their contents. Silver nanoparticles have a higher frequency plasmon resonance than gold particles, but the principles are otherwise the same. Soon after, Caruso and colleagues [51,52] used gold nanoparticles in a similar role. In this case, the illuminate-and-burst principle was successfully demonstrated by using a release of lysozyme to destroy the bacterium Micrococcus lysodeikticus. A schematic illustration of this latter scheme is shown in Figure 3. It was also shown that the capsules could be coated with a lipid bi-layer that was in turn functionalized with antibodies [51]. 


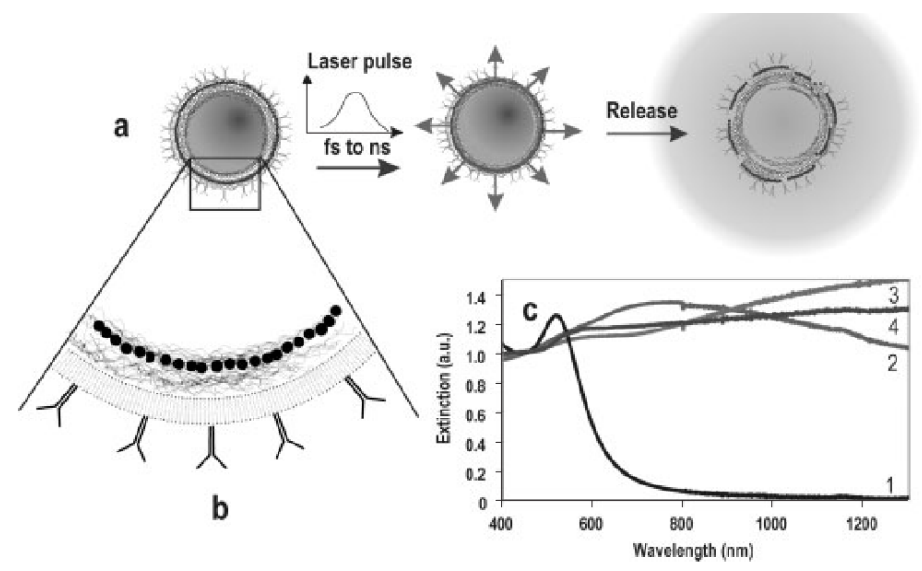

Figure 3. Controlled delivery of a chemical payload by a antibody-functionalized polymer capsule. Application of laser light causes the gold nanoparticles in the shell of the capsule to heat up, bursting the container (reproduced from Radt et al [62] with permission).

A related idea is to bind the drug to the outside of a gold nanoparticle, using for example a light-sensitive and conformation-changing molecule such as spyropyran [53] to hold it in place. When spyropyran is irradiated by $320 \mathrm{~nm}$ light (in the lowenergy ultraviolet part of the spectrum) it undergoes a conformation change from an open form to a closed form. The open form can form complexes with amino acids, but these complexes are destroyed and the amino acid released when the change to the closed form occurs [53]. Gold is the ideal choice for the substrate particle, as it provides a convenient chemistry for attachment, but in this case the optical properties of gold are not directly exploited. Targeting of such particles is also by passive means only but, once again, further localization of the effect can be achieved with the light source.

It has also been demonstrated that tumor necrosis factor (TNF) can be attached to gold nanoparticles and readily concentrated in murine tumors with beneficial results [47]. Once again, targeting is by extravasation and essentially passive in nature. No photoactivation of the payload was invoked in this particular work but it is obvious that plasmonic heating of the gold particles, if it had been attempted, would very likely have exerted an influence on the outcome.

An issue to be born in mind with these drug delivery schemes is that the volume of payload that can be carried by individual nanoparticles is very limited, and that in the absence of any external scheme to trigger release, the concentration of therapeutic agent released by gradual diffusion out, or breakdown of, the packaging might be too low to be effective [15] 


\section{Future developments}

Despite many advances in the field, there is a still a significant requirement for new technologies that will allow for the earlier treatment of diseases such as cancer, particularly if they also offer greater specificity and cost-effectiveness. It can be expected that continued development of the techniques described above will take place, particularly in respect of active targeting. Also, it is probable that the chemical and physical-based therapies reviewed above will be complemented by gene therapies delivered by nanoparticles.

It is apparent that gold nanoparticles have unique chemical and physical properties that facilitate and commend their use in these applications, despite some questions that remain unanswered (Box 2). In particular, their tunable optical properties and flexible surface chemistry suggest their use in photo-thermal therapeutic treatments. As discussed, the efficiencies of the process can be considerably enhanced by exploiting various passive targeting strategies to concentrate the nanoparticles in the tissue to be treated. In principle, active targeting of the nanoparticle is also possible, although further work on methods to evade the immune system en route to the target also needs to be considered.

\section{References}

1 Daniel, M.-C. and Astruc, D. (2004) Gold nanoparticles: assembly, supramolecular chemistry, quantum-size-related properties, and applications toward biology, catalysis, and nanotechnology. Chem. Rev.104, 293-346

2 Love, J.C. et al. (2005) Self-assembled monolayers of thiolates on metals as a form of nanotechnology. Chem. Rev. 105, 1103-1169

3 Cortie, M.B. (2004) The weird world of nanoscale gold. Gold Bull. 37, 12-19

4 Thanh, N.T.K. and Rosenzweig, Z. (2002) Development of an aggregation-based immunoassay for anti-protein a using gold nanoparticles. Anal. Chem. 74, 1624- 1628

5 Faulk, W.P. and Taylor, G. (1971) An immunocolloid method for the electron microscope. Immunochem. 8, 1081-1083

6 Elghanian, R. et al. (1997) Selective colorimetric detection of polynucleotides based on the distance-dependent optical properties of gold nanoparticles. Science 277, 1078-1081

7 Bendayan, M. (2001) Worth its weight in gold. Science 291, 1363-1365

8 Nam, J. et al. (2003) Nanoparticle-based bio-bar codes for the ultrasensitive detection of proteins. Science 301, 1884-1886

9 Hirsch, L.R. et al. (2003) A whole blood immunoassay using gold nanoshells. Anal. Chem. 75, 2377-2381

10 Zhao, X. et al. (2004) A rapid bioassay for single bacterial cell quantitation using bioconjugated nanoparticles. Proc. Natl. Acad. Sci. USA, 101, 15027-15032

11. Loo, C. et al. (2004) Nanoshell-enabled photonics-based imaging and therapy of cancer. Technology in Cancer Research and Treatment, 3, 33-40

12. Kelly, K.L. et al. (2002) The optical properties of metal nanoparticles: The influence of size, shape, and dielectric environment. J. Phys. Chem. B 107, 668-677 
13 Shaw III, C.F. (1999) Gold-based therapeutic agents. Chem Rev. 99, 2589-2600

14. Salata, OV. (2004) Applications of nanoparticles in biology and medicine. J. Nanobiotechnol. 2, 16

15. Moghimi, S.M. et al. (2005) Nanomedicine: current status and future prospects . FASEB 19, 311 330

16. Shum, P. (2001) Phototriggering of liposomal drug delivery systems. Adv.Drug Deliv. Rev. 53, 273284

17 Sershen, S.R. et al. (2000) Temperature-sensitive polymer-nanoshell composites for photothermally modulated drug delivery. J. Biomed. Mater. Res. 51, 293-298

18 Hirsch, L.R. et al. (2003) Nanoshell-mediated near-infrared thermal therapy of tumors under magnetic resonance guidance. Proc. Natl. Acad. Sci. USA, 100, 13549-13554

19 West, J.L. and Halas, N.J. (2003) Engineered nanomaterials for biophotonics applications: improving sensing, imaging, and therapeutic. Annu. Rev. Biomed. Eng. 5, 285-292

20. Pyykko, P. (2004) Theoretical chemistry of gold. Angew. Chem. Internl Ed. 43, 4412-4456.

21. O’Neal, D.P. et al. (2004) Photo-thermal tumor ablation in mice using near infrared-absorbing nanoparticles. Cancer Lett. 209, 171-176

22. Aden, A.L. and Kerker, M. (1951) Scattering of electromagnetic waves from two concentric spheres. J. App. Phys. 22, 1242-1246

23. Kerker, M. and Blatchford, C.G. (1982) Elastic scattering, absorption, and surface-enhanced Raman scattering by concentric spheres comprised of a metallic and a dielectric region. Phys. Rev. B 26, 4052-4063

24 Neeves, A.E. and Birnboim, M.H. (1989) Composite structures for the enhancement of nonlinearoptical susceptibility. J. Opt. Soc. Am. B 6, 787-796

25 Nedeljkovic, J. and Patel, R.C. (1991) Observation of plasmon-enhanced optical extinction in silvercoated silver bromide particles. Appl. Phys. Lett. 58, 2461- 2463

26 Zhou, H.S. et al. (1994) Controlled synthesis and quantum-size effect in gold-coated nanoparticles. Phys. Rev. B 50, 12052-1205

27. West, J.L. and Halas, N.J. (2000) Applications of nanotechnology to biotechnology. Curr. Opin. Biotechnol. 11, 215-217

28 Averitt, R.D. et al. (1997) Plasmon resonance shifts of $\mathrm{Au}$ coated $\mathrm{Au}_{2} \mathrm{~S} \quad$ nanoshells: insight into multicomponent nanoparticle growth. Phys. Rev. Lett. 78, 4217-4220

29 Oldenburg, S.J. et al. (1998) Nanoengineering of optical resonances. Chem. Phys. Lett. 288, 243247

30 Averitt, R.D. et al. (1999) The ultrafast optical properties of gold nanoshells. J. Opt. Soc. Amer. B 16, 1814-1823.

31 Oldenburg, S.J. et al. (1999) Infrared extinction properties of gold nanoshells. App. Phys. Lett. 75, 2897-2899

32 Pham, T. et al. (2002) Preparation and characterization of gold nanoshells coated with selfassembled monolayers. Langmuir 18, 4915-4920 
33 Jackson, J.B. et al. (2003) Controlling the surface enhanced Raman effect on the surface of a coreshell nanoparticle. App. Phys. Lett. 82, 257-259

34 Oldenburg, S.J. et al. (2002) Rice University. Metal nanoshells. U.S. Patent 6344272

35 West, J.L. et al. (2002) Rice University. Temperature-sensitive polymer/nanoshell composites for photothermally modulated drug delivery. U.S. Patent 6428811

36 West, J.L. et al. (2003) Rice University. Optically-active nanoparticles for use in therapeutic and diagnostic methods. U.S. Patent 6530944

37 West, J.L. et al. (2002) Rice University. Optically-absorbing nanoparticles for enhanced tissue repair. U.S. Patent 6685730

38 Oldenburg, S.J. et al. (2004) Rice University. Metal nanoshells. U.S. Patent 6685986

39 Caruso, F. (2001) Nanoengineering of particle surfaces. Adv. Mater. 13, 11-22

40 Graf, C. and van Blaaderen, A. (2002) Metallodielectric colloidal core-shell particles for photonic applications. Langmuir 18, 524-534

41 Jain R.K. and Booth M.F. (2003) What brings pericytes to tumor vessels? Journal of Clinical Investigation 112, 1134-1136

42 McNeil, S.E. (2005) Nanotechnology for the biologist. J. Leukoc. Biol. $\quad$ DOI:10.1189/jlb.0205074 (http://www.jleukbio.org)

43 Jolesz, F.A. and Hynynen, K (2002) Magnetic-resonance image-guided focused ultrasound surgery. Cancer J. 8, S100-S112

44 Peceros, K.E. et al. (2005) Dipole-dipole plasmon interactions in gold-on-polystyrene composites. $J$. Phys. Chem. B, DOI: 10.1021/jp0523470 (http://pubs.acs.org/journals/jpcbfk/).

45 Pitsillides, C.M. et al. (2003) Selective cell targeting with light-absorbing microparticles and nanoparticles. Biophys. J. 84, 4023-4032

46 Hainfeld, J.F. et al. (2004) The use of gold nanoparticles to enhance radiotherapy in mice. Phys. Med. Biol. 49, N309-N315

47 Paciotti, G.F. et al. (2004) Colloidal gold: a novel nanoparticle vector for tumor directed drug delivery. Drug Deliv. 11, 169-183

48 Glomm, W.R. (2005) Functionalized gold nanoparticles for application in biotechnology. J. Disp. Sci. Tech. 26, 389-414

49 Schmitz, V. (2002) Gene therapy for liver diseases: recent strategies for treatment of viral hepatitis and liver malignancies. Gut 50, 130-135

50 Skirtach, A.G. et al. (2004) Remote activation of capsules containing Ag nanoparticles and IR dye by laser light. Langmuir 20, 6988-6992

51 Radt, B. et al. (2004) Optically addressable nanostructured capsule. Adv. Mater. 16, 2184-2189

52 Angelatos, A.S. et al. (2005) Light-responsive polyelectrolyte/gold nanoparticle microcapsules. $J$ Phys. Chem. B 109, 3071-3076

53 Thomas, K.G., and Kamat, P.V. (2003) Chromophore-functionalized gold nanoparticles. Acc. Chem. Res. 36, 888-898 
54 Bohren, C.F. ; Huffman, D.R. Absorption and Scattering of Light by Small Particles; WileyInterscience: New York, 1998.

55 Norman, T.J. et al. (2002) Near infrared optical absorption of gold nanoparticle aggregates. J. Phys. Chem. $B$ 106, 7005-7012

56 Ung, T. et al. (2001) Optical properties of thin films of $\mathrm{Au} @ \mathrm{SiO}_{2}$ particles. J. Phys. Chem. B. 105, 3441-3452

57 Pérez-Juste, J. et al. (2005) Gold nanorods : Synthesis, characterization and applications, Coordination Chemistry Reviews 249, 1870-1901

58 Liu, J. et al. (2005) Fabrication of hollow metal 'nano-caps' and their red-shifted optical absorption spectra. Adv. Mater. 17, 1276-1281 


\section{BOX 1}

\section{Optical properties of metal nanoparticles}

A nanoscale electronic effect known as the plasmon resonance causes metallic nanoparticles to absorb and scatter electromagnetic radiation of wavelengths considerably larger than the particles themselves. These effects are particularly obvious in the visible part of the spectrum for gold, silver and copper. In the case of gold nanospheres the 'plasmon' resonance, and hence absorption, is at around $520 \mathrm{~nm}$ (corresponding to green light). In fact, a proportion of the incident light is also scattered, but it is the light that is absorbed that will cause heating in the particles. This results in a highly localized increase of temperature that is exploited in the various proposed photo-thermal therapeutic uses of gold nanoparticles. Further background information on the optical phenomena involved may be found in a standard text, such as that of Bohren and Huffman [54].

It may be convenient to shift the wavelength of maximum absorption into the near infrared because the body is more transparent at those wavelengths [11,21]. This may be achieved with gold nanoshells [17], or by enhancing the dipole-dipole interaction between aggregated gold nanospheres $[44,55,56]$, or by decreasing the symmetry of the particles to form, for example nanorods [57]. Gold nanorods have two plasmon absorption peaks, and the position of the second peak can be moved deep into the NIR by controlling the aspect ratio of the rod. Gold 'nano-caps' [58] have similar red-shifted extinction spectra. 


\section{BOX 2}

\section{Outstanding questions}

Although most available evidence suggests that colloidal gold particles are chemically inert in vivo, the issue is not definitively settled and requires proper, controlled study. Certainly, it is known that such particles will be ingested by cells of the immune system unless rendered 'invisible' by one of the so-called 'stealth' technologies, and the overall implications of this need to be considered. Of course removal of particles from the vasculature by leukocytes is especially problematic in respect of actively-targeted nanoparticles, which by definition need to expose some antigenic structure on their exteriors. Perhaps there will be a short period of opportunity available to such particles to be delivered to their target sites before the patient's immune system can mount a full reaction. Finally, one of the most interesting challenges is whether it will be feasible to actively target intra-cellular pathogens using functionalized nanoparticles, since the antigenic properties of these are usually well concealed. 